\title{
LXXXIV. Filices novae a cl. Dr. O. Buchtien in Bolivia collectae.
}

Von Dr. E. Rosenstock, Gotha.

(Originaldiagnosen.)

\section{V.1)}

54. Pteris Haenkeana Presl var, adaucta Ros,, nov, var. ( $P$. adaucta Ros. in sched.)

Varietas frondibus majoribus, ad $4 \mathrm{~m}$ longis, tripinnatis (pinnis basalibus ad $70 \mathrm{~cm}$ vel ultra longis, infra bipinnatis, sursum pinnatis. pinnis secundariis c. 12-jugis, inferioribus 2-3-jugis, triphyllis, ceteris diphyllis vel simplicibus, pinnis tertiariis basi saepe valde inaequalibus, ad $20 \mathrm{~cm}$ longis, $2 \frac{1}{2} \mathrm{~cm}$ latis a typo et a $P$. ampla Kze. diversa.

Bolivia: lungas septentrionalis, Polo-Polo prope Coroico, $900 \mathrm{~m}$ alt., Oct.- Nov. 1912, leg. Dr. 0. Buchtien no. 3546.

55. Asplenium tricholepis Ros., nov. spoc.

Euasplenium; rhizomate erecto, lignoso, apice dense paleaceo. pale is ferrugineis, tenuibus, lanceolatis, acuminatis, strictis, $2 \mathrm{~cm}$ vel paullo plus longis, $2 \frac{1}{2} \mathrm{~mm}$ basi latis, pilis articulatis, longis, tenuibus. flexuosis dorso sparse, margine densius ornatis; stipitibus fasciculatis. stramineo-viridibus, nudis, glaberrimis, ad $20 \mathrm{~cm}$ longis; lamin is $\theta$ bas, cuneata ovato-acuminatis, herbaceis, supra saturate viridibus, subtus pallidioribus, ad $34 \mathrm{~cm}$ longis, $16 \mathrm{~cm}$ latis, pinnatis; pinnis 2-4.jugis cum impari, alternis, erectis, strictis vel paullo incurvatis, summis sessilibus exceptis breviter petiolatis (petiolo lamina decurrente marginato), basalibus ad $18 \mathrm{~cm}$ longis, $3 \mathrm{~cm}$ latis, $\theta$ basi inaequaliter cuneata (anteriore adaucta, exauriculata) lanceolatis, acuminatis, apice obtuso, margine integerrimo, ceteris sursum paullo decrescentibus, terminali maximai ad $23 \mathrm{~cm}$ longa, $3 \frac{1}{2} \mathrm{~cm}$ lata; $\mathrm{rhachibus} \mathrm{compressis,} \mathrm{pinnis} \mathrm{decurrenti-}$ bus anguste marginatis; costis viridibus, supra immersis, subtus prominulis et sparse furfuraceis; venis plerisque bis vel pluries furcatis, ramo antico cujusque fasciculi sorifero; venis linearibus, parallelis, a costa et margine paullo remotis; indusio firmo, angusto.

Bolivia: Yungas septentrionalis, Polo-Polo prope Coroico, $900 \mathrm{~m}$ alt., (ict.-Nov. 1912, leg. Dr. 0. Buchtien no. 3330.

Diese Art gleicht habituell $\operatorname{dem} \boldsymbol{A}$. oligophyllum Klfs., unterscheidet sich aber von ihm und von den zur Gruppe des $A$. integerrimum Spr. gehörigen Arten sofort durch die grossen, dünnen, abor doch straffen Rhizomschuppen, sowie durch die Bekleidung dieser mit langen, weichen Haaren.

1) Fortsetzung der Artikel in Rep. V (1908), pp. 228-239); Rep. VI (1909), pp. 308-316, Rep. IX (1911), pp. 342-34t und Rep. XI (1912), pp. 53-60. 
56. Asplenium auricularium Desv, var. acutidens Ros., nov. var.

Varietas dentibus acutis, in lobulis inferioribus lateris anterioris plerumque binis a typo diversa.

Bolivia: Yungas septentrionalis, Polo-Polo prope Coroico, $900 \mathrm{~m}$ alt., Oct. - Nov. 1912, leg. Dr, O. Buchtien no. 3340.

Die Fiedern des typischen $A$, auricularium Desv. aus Brasilien, mit dem die als $A$. lunulatum Sw. ausgegebenen Exemplare der no. 147 meiner Filices austro brasilienses exsiccata $\theta$ gut übereinstimmen, sind „aequaliter obtuse dentatae" (Desv.).

57. Asplenium auricularium Desv. var. subintegerrima Ros., nov. var.

Varietas pinnis plerisque inferioribus, saepius et medialibus reflexis, ceteris horizontaliter patentibus, omnibus levissime crenato-dentatis, dentibus oculo nudo vix conspicuis a typo diversa. Folia ad $50 \mathrm{~cm}$ longa, $4 \mathrm{~cm}$ lata.

Bolivia: Yungas septentrionalis, Polo.Polo prope Coroico, $900 \mathrm{~m}$ alt., Oct.-Nov. 1912, leg. Dr. O. Buchtien no. 3338.

58. Asplenium discrepans Ros., nov. spec.

Euasplenium; rhizomate erecto, paleis parvis, hyalinis, anguste lanceolatis, integerrimis apice obtecto; stipitibus fasciculatis, divergenti-flexuosis, brevibus $(1-2 \mathrm{~cm}$ longis), cum $\mathrm{rhachibus}$ griseo-viridibus, antice sulcatis, glabrescentibus; laminis elongato-oblongis, breviter acuminatis, glancescenti-viridibus, herbaceis, glaberrimis, ad $12 \mathrm{~cm}$ longis, $3 \mathrm{~cm}$ latis (plerumque angustioribus), pinnatis; pinnis ad 24 utrinque, alternis, appropinquatis, breviter petiolatis, basalibus vix reflexis, proximis horizontalibus, superioribus erectis, medialibus maximis ad $1 \frac{1}{2} \mathrm{~cm}$ longis, $5 \mathrm{~mm}$ latis, e basi asymmetrica, superiore truncata et obsolete auriculata, inferiore anguste cuneata, trapezio-oblongis, obtusis, margine anteriore infra bidentato, supra cum exteriore simpliciter dentato, margine posteriore infra usque ad mediam vel paullo ultra integerrimo, sursum dentibus paucis simplicibus, levibus instructo; pinnis inferioribus paullo abbreviatis vel proximis subaequalibus, superioribus sensim diminutis; venis indistinctis, plerisque furcatis; soris linearibus, costa $\theta$ magis quam margini appropinquatis, maturis subconfluentibus; in dusio membranaceo, integerrimo.

Bolivia: Yungas septentrionalis, Polo-Polo prope Coroico, $900 \mathrm{~m}$ alt., Oct.-Nov. 1912, leg. Dr. O. Buchtien no, 3341.

Eine bezüglich der Gestalt der Fiedern zwischen $A$. auricularium Desv. und $A$. pulchellum Raddi stehende, niedrige, wenig charakteristische Art, mit auseinandergespreizten Blättern.

59. Asplenium poloënse Ros., nov. spec.

Euasplenium; rhizomate erecto, paleis anguste lanceolatis, membranaceis, parvis apice obtecto; stipitibus fasciculatis, $2-4 \mathrm{~cm}$ longis, tenuibus, cum rhachibus flexuosis, griseo-viridibus, antice sulcatis, glabris; laminis elongato-oblongis, acuminatis, laete viridibus, herbaceis, glaberrimis, ad $15 \mathrm{~cm}$ longis, $3-4 \mathrm{~cm}$ latis, pinnatis; pinnis usque ad 18 fere utrinque, alternis, subappropinquatis, breviter petiolatis, basalibus 
vix reflexis, medialibus et superioribus erecto-patentibus; medialibus maximis ad $3 \mathrm{~cm}$ longis, basi $8 \mathrm{~mm}$, medio $3-4 \mathrm{~mm}$ latis, $\theta$ basi asymmetrica, superiore truncata, cum rhachi parallela, distincte auriculata, inferiore anguste cuneata, trapezio-oblongis, acutis vel acuminatis, saepe incurvatis, margine anteriore profunde inciso-lobato (lobis inferioribus 2(-3) bidentatis), posteriore, dentibus 1-3 infraapicalibus, simplicibus exceptis, integerrimo; pinnis inferioribus paucis abbreviatis, superioribus gradatim diminutis, in apicem grosse dentatum sensim transeuntibus; ve $n$ is auriculae bis bifurcatis, loborum 1-2 sequentium furcatis, ceteris simplicibus; soris linearibus, lobos intrantibus; indusio membranaceo, integerrimo.

Bolivia: Yungas septentrionalis, Polo-Polo prope Coroico, $900 \mathrm{~m}$ alt., Oct.-Nov. 1912, leg. Dr. O. Buchtien no. 3345.

Wie die vorige Art mit $A$. pulcherrimum Raddi verwandt, durch die stark geöhrten und tief eingeschnittenen Fiedern jedoch dem $A$. Fernandezianum Kze. sich nähernd.

60. Asplenium abscissum Willd. var. subaequilateralis Ros., nov. var.

Varietas pinnis inferioribus ac medialibus basi subaequaliter angustatis, basi posteriore convexa nec exciso-cuneata a typo diversa.

Bolivia: Yungas septentrionalis, Polo-Polo prope Coroico, $900 \mathrm{~m}$ alt, Oct.-Nov. 1912, leg. Dr. O. Buchtion no. 3349.

61. Asplenium dimidiatum Sw. var. boliviensis Ros., nov. var.

Varietas pinnis angustioribus, plerisque exauriculatis, sursum decrescentibus et in lobos apicis sensim transeuntibus a typo diversa.

Bolivia: Yungas septentrionalis, Polo.Polo prope Coroico, $900 \mathrm{~m}$ alt., Oct.-Nov. 1912, leg. Dr. O. Buchtien no. 3333.

62. Diplazium cuneifolium Ros., nov. spec.

Eudiplazium; rhiz omate erecto, lignoso, paleis atrobrunneis, lanceolatis, ad $1 \mathrm{~cm}$ longis, $3 \mathrm{~mm}$ latis, sparse glanduligeris apice obtecto; stipitibus fasciculatis, erectis, e basi fuscescenti cum rhachibus viridi-stramineis, leviter puberulis, c. $30 \mathrm{~cm}$ longis, $3 \mathrm{~mm}$ medio crassis; laminis oblongo-lanceolatis, acuminatis, c. $50 \mathrm{~cm}$ longis, $24 \mathrm{~cm}$ latis, pinnatis, herbaceis, supra olivaceo-viridibus, praeter costam glaberrimis, nitidis, subtus paullo pallidioribus, opacis, ad costas venasque pilis uni- vel paucicellularibus, albidis vel ferrugineis obsessis; pinnis 15-20-jugis, subrecte patentibus, subalternis. usque ad $3 \mathrm{~cm}$ inter se remotis, breviter petiolatis, e basi subaequaliter cuneata elongato-oblongis, in apicem sensim angustatum acuminatis, in lobos obtusos, integerrimos, $1 / 3$ laminae dimidjatae longitudine non excedentes incisis, medialibus maximis ad $13 \mathrm{~cm}$ longis, $2 \mathrm{~cm}$ latis, basalibus usque ad $10 \mathrm{~cm}$ fere longitudine abbreviatis, superioribus sensim in lobos apicis pinnatifidi transeuntibus; venis pinnatis; venulis ad $4-5$ utrinque, simplicibus; soris linearibus, e costula usque fere ad marginem productis, basalibus anticis diplazioideis; indusio membranaceo, puberulo, margine fimbriato.

Bolivia: Yungas septentrionalis, Polo-Polo prope Coroico, $900 \mathrm{~m}$ alt., Oct.-Nov. 1912, leg. Dr, O, Buchtien no. 3390. 
Diese Art steht dem Diplazium Ottonis Kl. am nächsten, das sich durch rechtwinklig gestutzte Fiederbasis, mehr geradlinigen Vorderrand der Segmente und kahle Blattunterseite hinreichend unterscheidet.

63. Diplazium divergens Ros., nov. spec.

Eudiplazium; (rhizoma, stipites?); l amin is amplis, bjpinnato-pinnatifidis, subcoriaceo-chartaceis, inter venas glaberrimis, supra saturate viridibus, subtus pallidioribus; $\mathrm{rhachibus} \mathrm{costisque} \mathrm{viridulis,} \mathrm{fibrilloso-}$ squamulosis, glabrescentibus, costulis venisque supra glaberrimis, subtus paleolis lineari-lanceolatis, fibrillosis adspersis; pinnis primariis alternis, petiolis c. $4 \mathrm{~cm}$ vel ultra longis instructis, patentibus, oblongolanceolatis, acuminatis, ad $50 \mathrm{~cm}$ longis, $18 \mathrm{~cm}$ latis; pinnis secundariis c. 14.jugis, alternis, subdistantibus, praeter summas sessiles vel adnatas petiolis $3-4 \mathrm{~mm}$ longis suffultis, medialibus maximis ad $9 \mathrm{~cm}$ longis, $2 \frac{1}{2} \mathrm{~cm}$ latis, e basi truncata lineari-lanceolatis, in apicem acuminatum anguslatis, profunde pinnatifidis, basalibus proximis paullo minoribus, superioribus gradatim diminutis, in apicem lobatum sensim transeuntibus; lobis linearibus, obtusis, margine revoluto obsolete crenatis, recte patentibus vel superioribus paullo obliquis; venis ad 7-8 utrinque, distantibus, erecto-paientibus, simplicibus; soris basalibus anticis diplazioideis, c. $2 \mathrm{~mm}$ longis, ceteris simplicibus, $1 \mathrm{~mm}$ longitudinis vix excedentibus, omnibus costulae valde appropinquatis; indusiis magnis, membranaceis, albidis.

Bolivia: Yungas septentrionalis, Polo-Polo prope Coroico, $1100 \mathrm{~m}$ alt., Oct.-Nov. 1912, leg. Dr. O. Buchtien no. 3393.

Die Art unterscheidet sich von dem ihr nahestehenden D. hians Kze. durch langgestielte Fiedern, sowie durch breitere und linear-lanzettliche Fiederchen. Auch ist die Textur derber, die Farbe heller und die sori sind kürzer als bei der genannten Art.

64. Dryopteris tristis (Kze.) C. Chr. var. auriculata C. Chr. et Ros., nov. var.

Varietas pinnis 4-5 jugum inferiorum basi parum angustatis, auricula usque ad $9 \mathrm{~mm}$ longa antice instructis, costulis pinnarum inferiorum ac mediarum anadromis, superiorum 3-4 jugum modo homodromis vel anadromis a typo diversa.

Bolivia: Yungas septentrionalis, Polo-Polo prope Coroico, $1100 \mathrm{~m}$ alt., Oct.-Nov. 1912, leg. Dr. O. Buchtien no. 3433.

65. Dryopteris leucothrix C. Chr. var. glanduligera C. Chr. et Ros., nov. var.

Varietas stipite deorsum pilis longis, albis, mollibus lanoso, facie frondis inferiore glandulosa nec (costis exceptis) pilosa, indusiis glandulis aureis instructis, minus dense pilosis, maturis evanescentibus a typo diversa.

Bolivia: Yungas septentrionalis, Polo-Polo prope Coroico, $900 \mathrm{~m}$ alt., Oct.-Nov. 1912, leg. Dr. O. Buchtien no. 3407. 
66. Dryopteris ptarmiciformis C. Chr, et Ros., nov. spec.

Lastrea; rhizomate erecto, paleis lanceolatis, ferrugineo-brunneis, margine tergoque pilosulis vestito; stipitibus dense fasciculatis, basi nigrescente, paleacea excepta stramineis, nudis, in sulco hirtis, ceterum pilis paucis brevibus vestitis vel glabrescentibus, usque ad pinnas normales c. $12 \mathrm{~cm}$ longis, $1 \frac{1}{3} \mathrm{~mm}$ crassis; laminis deorsum obsoletis, pinnis inferioribus $3-4$ jugis ad meras auriculas reductis vel glandiformibus, sursum elongato-ovalibus, acuminatis, herbaceis, laete viridibus, in facie superiore pilis albidis subulatis prope marginem adspersis, utrinque obscure albido-glandulosis vel furfuraceis, c. $20 \mathrm{~cm}$ longis, $10 \mathrm{~cm}$ latis, pinnato-pinnatifidis; pinnis (non reductis) c. 12 -jugis, brevissime petiolatis, iis inferiorum $1-2$ jugum plerumque reflexis, ceteris erecto-patentibus, strictis, medialibus maximis c. $6 \mathrm{~cm}$ longis, vix $1^{1 / / 2} \mathrm{~cm}$ latis, e basi subaequali lineari-lanceolatis, breviter acuminatis, usque fere ad costam inciso-lobatis; inferioribus parum abbreviatis, infimis - transitum ad pinnas auriculiformes formantibus - ad $1 \mathrm{~cm}$ longitudinis repente reductis; superioribus gradatim diminutis, in lobos apicis demum serrati vel subintegri sensim transeuntibus; segmentis densis, linearibus, parallelis, valde obliquis, obtusis vel oblique acutis, c. $8 \mathrm{~mm}$ longis, $2^{1 / 2} \mathrm{~mm}$ latis, basalibus binorum jugum inferiorum paullo diminutis, ceterorum proximis aequalibus; rhachibus antice dense strigoso-hirtis, postice pilis patentibus brevibus (paucis longioribus intermixtis) puberulis; costis supra breviter strigosis, subtus cum costulis glabris; nervis lateralibus simplicibus, pellucidis, c. 6-jugis; soris margini appropinquatis; indusio firmulo, persistente, margine breviter glandulosofimbriato, ceterum glaberrimo.

Bolivia: Yungas septentrionalis, Polo.Polo prope Coroico, $900 \mathrm{~m}$ alt., Oct.-Nov. 1912, leg. Dr. O. Buchtien no. 3545 .

Die mit $D$. oligocarpa verwandte Art unterscheidet sich von dieser durch deutliches Indusium, grössere Kahlheit der ganzen Pflanze, besonders des Parenchyms, sehr schräg gestellte Segmente, durch die sie sich im Aussehen der $D$. ptarmica (Kze.) nähert, und durch plötzlich verkürzte untere Fiedern. Dieselbe Pflanze erhielt ich aus Brasilien von Petropolis bei Rio de Janeiro (l. Th. Schnapp no. 51 p.; 1904).

67. Dryopteris subandina C. Chr. et Ros., nov. spec.

Lastrea; rhizumate breviter repente, demum suberecto, paleis ferrugineis, membranaceis, flaccidis, ovato-lanceolatis, breviter acuminatis, glabris, integerrimis vel dentes paucos glanduligeros ciliasve $1-2$ gerentibus instructo; stipitibus fasciculatis (ad 6 in specimine), e basi flexuosa, nigricante, paleacea erectis, stramireis vel ochraceis, nitidis, nudis, pilis brevibus albidis, mollibus praesertim in sulco adspersis, c. $20 \mathrm{~cm}$ (usque ad pinnas normales) longis, $3 \mathrm{~mm}$ fere supra basim crassis; laminis elongato-oblongis, utroversus (deorsum maxime) acuminatis, pinnato-pinnatifidis, subcoriaceis, supra laete viridibus, subtus pallidioribus, pilis albidis, subulatis, strictis vel subhamatis ad nervos et in parenchymate utrinque vestitis glandulisque aureis facie inferiore 
hinc inde ornatis, ad $70 \mathrm{~cm}$ longis, $18 \mathrm{~cm}$ medio latis; pinnis numerosis, sessilibus, subop positis, subapproximatis, subrecte patentibus, strictis vel subflexuosis, medialibus maximis ad $9 \mathrm{~cm}$ longis, $13 / 4 \mathrm{~cm}$ infra latis, e basi truncata, subaequali vel paullo utrinque adaucta lanceolatis, acuminatis; inferioribus gradatim diminutis et remotioribus, reflexis, demum auriculiformibus vel imis glandiformibus; superioribus sensim abbreviatis et simplicioribus, in lobulos apicis brevi, pinnatifidi transeuntibus; segmentis ad 28 utrinque, paullo obliquis, linearibus, acutiusculis, margine revoluto, subintegerrimo, inflmis c. $8 \mathrm{~mm}$ longis, $4 \mathrm{~mm}$ latis, inde sursum gradatim diminutis, in dentes apicis acuminati, serrati sensim transeuntibus; rhachibus costisque utrinque (infra densius) breviter hirtis; venis lateralibus simplicibus, utrinque ad $9-10$; 'soris supramedialibus, contiguis, maturis subconfluentibus; sporangiis nudis vel seta decidua hinc inde instructis; indusiis parvis, setis longis, albidis dense ornatis.

Bolivia: Cataña ad fl. llimano, secus fossas, $2550 \mathrm{~m}$ alt., Nov. 1911 , leg. Dr. 0. Buchtien no. 3120. - Forma minor ibid. no. 3119.

In der Blattgestalt kommt diese Art der Dryopteris supina (Sod.) und D. pachyrhachis (Kze.) nahe. Sie unterscheidet sich von beiden durch breitere Segmente, das Vorkommen von bewehrten Sporangien und durch die mit langen, weissen Borsten dicht bekleideten Indusien. Durch letztere ist sie auch von der ebenfalls nahestehenden $D$. concinna (Willd.) sicher zu unterscheiden.

68. Dryopteris nephrodioides (Kl.) Hieron. var. glandulosa C. Chr. et Ros.. nov. var.

Varietas magnitudine, textura, indumento stellato cum var. Biolleyi (Christ) congruens, sed glandulis faciem inferiorem ornantibus ab ea et ab omnibus $\S$ Goniopteridis speciebus adhuc notis diversa.

Bolivia: Yungas septentrionalis, Polo Polo prope Coroico, $900 \mathrm{~m}$ alt., leg. Dr. O. Buchtien no. 3410 .

69. Polypodium bolivianum Ros. var. brevipes Ros., nov. var.

Varietas stipitibus multo brevioribus $\left(1 \frac{1}{2}-3 \mathrm{~cm}\right.$ longis), pinnis inferioribus numerosis sensim decrescentibus, laminis utrinque sparse puberulis a typo diversa.

Bolivia: Yungas septentrionalis, Polo Polo prope Cornico, $900 \mathrm{~m}$ alt., log. Dr. O. Buchtien no. 3497.

70. Polypodium rhizocaulon Willd. var. hirsutula Ros., nov. var.

Varietas rhachibus laminisque utrinque pilis mollibus albidis lanoso. hirsutis a typo diversa.

Bolivia: Yungas septentrionalis, Polo Polo prope Coroico, $900 \mathrm{~m}$ alt., leg. Dr. O. Buchtien no. 3511.

71. Polypodium poloënse Ros., nov. spec.

Campyloneuron; rhizomate brevi, repente, paleis rigidiusculis, brunneis, ovato-lanceolatis, acuminatis vestito; stipitibus densis, vix $1 \frac{1}{2} \mathrm{~cm}$ longis, lamina decurrente anguste marginatis, cum costa mediana antice viridulis, postice brunnescentibus; laminis elongato-oblongis, utro. 
versus breviter angustatis, obtusis, margine revoluto, obsolete repando vel integro, subcoriaceo-herbaceis, subglaucescenti-viridibus, anguste albido-marginatis, glaberrimis, ad $20 \mathrm{~cm}$ longis, $3 \mathrm{~cm}$ latis; costulis cum venis crassitie et colore congruis, utrinque parum prominulis, subtus colore opaco conspicuis, maculis inter costam medianam et marginem plerumque ternis, costali valde elongata, radio e costula oriunte longo, libero. sequenti radiis binis liberis, hinc inde more Phlebodii conjunctis, instructa, vel uti tertia in maculas binas tertiarias, radios solitarios includentibus, divisa; soris paracostalibus lineam costae parallelam for$m$ antibus, ceteris irregulariter sparsis.

Bolivia: Yungas septentrionalis, Polo Polo prope Coroico, $900 \mathrm{~m}$ alt., leg. Dr. O. Buchtien no. 3525 .

Eine durch ihren kurzen Stiel und die nach oben und unten kurz verschmälerte Blattfläche auffallende Art der Campyloneuron Suruppe, mit breiten, sich auf der Unterseite der Blattfäche durch dunkle Schattierung ibzeichnenden Nerven.

72. Polypodium nitidissimum Mett. var. latior Ros., nov. var.

Varietas laminis latioribus, ad $70 \mathrm{~cm}$ longis, $15 \mathrm{~cm}$ latis, margine leviter undulatis, maculis inter costam et marginem ad 16-seriatis a typo diversa.

Bolivia: Yungas septentrionalis, Polo Polo prope Corojco, $900 \mathrm{~m}$ alt., leg. Dr. O. Buchtien no. 3526.

73. Elaphoglossum Bollivianii Ros., nov. spec.

Euelaphoglossum; rhizomate longe repente, phyllopodiis fuscescentibus, densis paleisque ferrugineis, membranaceis, flaccidis, lanceolatis, sparse fimbriatis, in apicem fibrillosum desinentibus dense ornato; stipitibus numerosis, c. ${ }^{1 / 2} \mathrm{~cm}$ inter se remotis, gracilibus, erectis, stramineis, paleolis minutis, adpressis, ovatis, breviter fimbriatis paucisque majoribus, ovalibus, subpatentibus adspersis; foliis sterilibus c. $20 \mathrm{~cm}$, fertilibus c. $14 \mathrm{~cm}$ longis; laminis sterilibus lineari-lanceolatis, utroversus longe et aequaliter angustatis, margine subreflexo, integerrimo, angustissime pallide marginato, tenuibus, membranaceis, pallide viridibus, paleolis minutis (supra rarioribus) utrinque adspersis; nervis tenuibus, plerisque furcatis, apice subincrassato; laminis fertilibus elongatooblongis, obtusis vol acutis, c. $12 \mathrm{~cm}$ longis, $2 \mathrm{~cm}$ latis; sporis ellipsoideis, reticulato-episporiatis, crenato-cristatis.

Bolivia: Yungas septentrionalis, Polo Polo prope Coroico, $900 \mathrm{~m}$ alt., leg. Dr. O. Buchtien no. 3461.

Eine sehr lang- und schmalblättrige Form der conforme-Gruppe (?), ron dünner Textur und mit kürzerem fertilen Blatt.

74. Elaphoglossum interruptum Ros., nov. spec.

Euelaphoglossum; rhizomate tenui, $2 \mathrm{~mm}$ vix crasso, repente, paleis ferrugineis, membranaceis, flaccidis, c. $6 \mathrm{~mm}$ longis, $1 \mathrm{~mm}$ latis, anguste lanceolatis, acuminatis, margine dentatis (dentibus brevibus, saepissime reflexis) sparseque ciliatis obtecto; stipitibus $1 / 2-1 \mathrm{~cm}$ distantibus, gracilibus, $2-5 \mathrm{~cm}$ longis, vix $1 \mathrm{~mm}$ crassis, stramineis vel brunnes- 
centibus, nudis, glaberrimis; laminis sterilibus ad $20 \mathrm{~cm}$ longis, $2 \mathrm{~cm}$ latis, elongato-oblongis, utroversus acuminatis, basi ima subito angustata vel plerumque interrupta, margine subintegerrimo, tenuibus, nudis, glaberrimis, utrinque viridibus; $\mathrm{n}$ ervis tenuibus, subpatentibus, apice valde incrassatis. Laminis fertilibus deficientibus.

Bolivia: Yungas septentrionalis, Polo Polo prope Coroico, $900 \mathrm{~m}$ alt., leg. Dr. O. Buchtien no. 3458.

Das Blatt ist sehr auffallend durch eine, wie es scheint, konstante Einschnürung der Basis, die meist so weit geht, dass ein kurzes Stück der Lamina von dem übrigen Teil völlig getrennt wird. Die Nervatur erinnert durch die stark verdickten Nervenenden an $E$. brachyneuron (Fée).

75. Elaphoglossum Buchtienii Ros., nov. spec.

Euelaphoglossum; rhizomate breviter repente, lignoso, $1 / 2 \mathrm{~cm}$ crasso, paleis lineari-lanceolatis, longe acuminatis, margine crebre et longe ciliatis, flaccidis, crispatis, ochraceis, brunnescenti-maculatis dense obtecto; stipitibus approximatis, phyllopodiis c. $2 \mathrm{~cm}$ longis, atrobrunneis, paleaceis suffultis, stramineis vel rubicundis, nudis, glaberrimis, stipitibus, foliorum sterilium c. $20 \mathrm{~cm}$ longis, $2 \mathrm{~mm}$ crassis, fertilium paullo longioribus; laminis sterilibus c. $28 \mathrm{~cm}$ longis, $8 \mathrm{~cm}$ latis, e basi late cuneata, ima breviter acuminata ovali-lanceolatis, breviter acuminatis, margine reflexo, albido, integerrimo, utrinque glaucescenti-viridibus, subnitidis, subcoriaceis, paleolis minutis, ciliatis, ochraceis, centro aterrimis adspersis, supra nudescentibus; costa mediana supra canaliculata, viridi.straminea, subtus rubidula, parum prominente, nuda; nervis subrecte patentibus, plerisque unifurcatis, paucis bifurcatis vel simplicibus, liberis, rarissime anastomosantibus, apice incrassato ante marginem cartilagineum desinentibus; laminis fertilibus ad $23 \mathrm{~cm}$ longis, $3 \mathrm{~cm}$ latis, e basi breviter cuneata elongato-oblongis vel linearibus, acuminatis vel acutis, nervis in facie superiore prope costam rubro-scalpturatis, ceterum aegre conspicuis, plerisque bifurcatis raro anastomosantibus, apicibus liberis; sporis ovalibus, vel subrotundis, inaequaliter crenulato-cristatis.

Bolivia: Yungas septentrionalis, Polo Polo prope Coroico, $900 \mathrm{~m}$ alt, leg. Dr. 0. Buchtien no. 3453 .

Das breitovale, scharf zugespitzte sterile Blatt entspricht dem Typus der Conformia, während das kurze, am Ende mit weichen, krausen Schuppen dicht besetzte Rhizom die Zugehörigkeit zur latifoliumGruppe bedingt.

76. Elaphoglossum Brausei Ros., nov. spec.

Hymenodium; rhizomate breviter repente, demum erecto, lignoso, crasso, apice dense paleaceo, paleis c. $7 \mathrm{~mm}$ longis, $2 \mathrm{~mm}$ vel paullo ultra latis, ochraceo-brunneis, subrigide membranaceis, ovato-lanceolatis, fibrilloso-acuminatis, margine ciliis longis flexuosis, lanceolato-linearibus, e cellularum seriebus $2-3$ efformatis, apice incrassatis ornatis; stipitibus approximatis, phyllopodiis perbrevibus, paleaceis suffultis, ochraceo- 
stramineis, paleolis minutis, dilacerato-ciliatis, adpressis paucisque majoribus ovato-lanceolatis, patulis, deciduis instructis, foliorum sterilium c. $50 \mathrm{~cm}$ longis, $1 / 2 \mathrm{~cm}$ medio crassis; laminis sterilibus c. $70 \mathrm{~cm}$ longis, $14 \mathrm{~cm}$ latis, e basi asymmetrica, altera cuneata, altera plus minusve rotundata elongato-oblongis, in apicem lanceolatum, subacuminatum desinentibus, margine cartilagineo, lutescenti-byalino, reflexo, repandulo, coriaceo-chartaceis, supra saturate viridibus, subtus pallidioribus, paleolis ferrugineis adpressis, minutis, ciliatis utrinque adspersis: costa mediana ochracea, supra profunde canaliculata, glabra, subtus convexa et paleis ferrugineis, fibrillosis adspersa; nervis e basi prope costam furcata pluries furcatis, intra costam et marginem pluries anastomosantibus, apicibus plerumque liberis, utrinque parum prominulis et aegre conspicuis; laminae fertilis pars superior tantum adest, nervis faciei anterioris plane conspicuis, rubello-scalpturatis, creberrime anastomosantibus; sporis reticulatis et crenato-cristatis.

Bolivia: Yungas septentrionalis, Polo Polo prope Coroico, $900 \mathrm{~m}$ alt., leg. Dr. 0. Buchtien no. 3447.

Diese grosse Art der latifolium-Gruppe gleicht habituell dem Elapho. glossum Hieronymi (Sodiro), auch stimmt die anastomosierende Aderung bei beiden ziemlich überein. Die Rhizomschuppen des letzteren sind jedoch schmäler, hellbraun und kurz bewimpert und die kleinen Schüppchen del Achsen und Blattflächen dunkel. Auch zeigt das fertile Blatt auf seiner Oberseite nicht die dunkel hervortretenden Nerven wie unsere Art.

77. Elaphoglossum erinaceum (Fée) Ros. var. boliviensis Ros., nov. var.

Varietas paleis rhizomatis quam in typo minoribus $(1 \mathrm{~cm}$ longis, $1 \mathrm{~mm}$ latis), crispatis et fibrilloso-acuminatis, margine hinc inde dentatis, paleis stipitum costae et marginis crebrioribus, brevioribus (ad $6 \mathrm{~mm}$ longis), aterrimis, e basi dilatata repente (nec sensim) setaceis, laminis versus apicem latioribus, demum breviter acuminatis. basi subrotundatis (nec cuneatis), stipitibus foliorum fertilium quam laminae pro ratione longioribus a typo Féei quadalupensi diversa.

Foliis utrisque (cum stipitibus) c. $42 \mathrm{~cm}$ longis, laminis sterilium ad $32 \mathrm{~cm}$ longis, $5 \frac{1}{2} \mathrm{~cm}$ latis, fertilium ad $17 \mathrm{~cm}$ longis, $2 \frac{1}{2} \mathrm{~cm}$ latis.

Bolivia: Yungas septentrionalis, Polo Polo prope Coroico, $900 \mathrm{~m}$ alt., leg. Dr. O. Buchtien no. 3470.

78. Elaphoglossum blandum Ros., nov. spec.

Euelaphoglossum; rhizomate repente, lignoso, c. $5 \mathrm{~mm}$ crasso, pale is pallide ferrugineis, membranaceis, c. $5 \mathrm{~mm}$ longis, $11 / 2 \mathrm{~mm}$ latis, lanceolatis, margine sparsim dentatis dense obtecto; stipitibus aggregatis, cum costa mediana pallide viridibus, paleis membranaceis, subadpressis, primum lacteis, serius lutescentibus, ovatis vel ovato-lanceolatis, acutis, dense fimbriato-dentatis, ad $5 \mathrm{~mm}$ longis, $3 \mathrm{~mm}$ latis vestitis; laminis sterilibus e basi brevi-cuneata elongato-ovalibus, valde obtusis, subcoriaceo-herbaceis, subglauco-viridibus, paleaceis, paleis albidis, membranaceis, ovato-lanceolatis, acutis vel rotundatis, ad costas 
Georg Bitter: Utber verschiedene Varietäten der Polylepis australis. 477

et secus marginem densioribus, in facie superiore demum subevanescentibus utrinque adspersis; nervis furcatis, immersis. Folia fructigera adhuc desiderantur.

Bolivia: Yungas septentrionalis, Polo Polo propo Coroico, $900 \mathrm{~m}$ alt., leg. Dr. 0. Buchtien no. 3456.

Die zur muscosum-Gruppe gehörige Art gleicht in der Blattgestalt dem $E$. Bellermannianum (Kl.), ist aber durch dünno Textur und die Farbe sowohl der Schuppen als der Blattflächen von ihm verschieden.

79. Elaphoglossum Orbignyanum (Fée) var. tectiformis Ros., nov. var.

Varietas Jaminis anguste lanceolatis, sensim acuminatis, c. $30 \mathrm{~cm}$ longis, $1 \frac{1}{2} \mathrm{~cm}$ latis, minus dense paleaceis a typo diversa.

Bolivia: Yungas septentrionalis, Polo Polo prope Coroico, $900 \mathrm{~m}$ alt., leg. Dr. O. Buchtien no. 3457.

\section{LXXXV. Überverschiedene Varietäten der Polylepis australis.}

Von Georg Bitter.

(Originalarbeit.)

Polylepis australis Bitt. in Englers Botan. Jahrb. 45 (1911), 619.

Ich hatte bereits in meiner "Revision der Gattung Polylepis" (Englers Bot. Jahrb., Bd. 45) auf den ausserordentlichen Polymorphismus dieser den Süden des Verbreitungsgebietes der Gattung einnehmenden Art hingewiesen; durch neuerliche Übersendung von Materialien aus dem nördlichen Argentinien seitens der Herren Teodoro Stuckert in Genf und Dr. M. Lillo in Tucumán zur Bestimmung lernte ich noch mehr Formen dieser interessanten Pflanze kennen. Besondere Beachtung ist einer eben dem Keimlingsstadium entwachsenen kleinen Rosettenpflanze von Oyada, Sierra de Achala, Prov. Córdoba (Stuckert no. 21 135!) zu schenken, bei der besonders die langen, dünnen, einzelligen, weissen Haare an Blattstiel, Rhachis und den Rändern der Blättchon auffallen. Die Pflanze hat in diesem Zustande habituell eine ziemlich grosse Ähnlichkeit mit manchen Acaena-Arten. An der Rückseite der jungen Blätter sowie an einem noch wenig gestreckten Stengelstück bemerkt man auch ziemlich dicht stehende, kurze, mehrzellige Haare mit dünnen Wandungen.

Von den übrigen, mir aus der Provinz Córdoba vorgelegenen Exemplaren sei folgendes bemerkt:

1. Stuckert no, 20638: Estancia Pampa San Luis, Sierra de Achala $2000 \mathrm{~m}$, sowio

2. Stuckert no. 21205: Oyada, Sierra de Achala sind $P$. australis Bitt. var. glabra (0. K.) Bitt.,

3. Stuckert no. 21720: Estancia Pampa de San Luis, Sierra de Achala = Polylepis australis Bitt., forma ad var. bijuga Bitt. vergens, sed foliis majoribus; 